\title{
Cuidando del embarazo: el caso de las parteras Tseltales en Chiapas, México
}

Caring for pregnancy: the case of Tseltal midwives in Chiapas, Mexico

\section{Cuidar da gravidez: o caso das parteiras Tseltal em Chiapas, no México}

\author{
Erasmo Jiménez López ${ }^{1}$, Gandhy Ponce Gómez²
}

\begin{abstract}
1Maestro en Enfermería. Universidad Nacional Autónoma de México. Jurisdicción Sanitaria Ocosingo. Responsable de Salud Perinatal. Chiapas, México. Correo electrónico: Erasmo.jimenez@yahoo.com.mx

2 Profesor de Carrera Titular A. Universidad Nacional Autónoma de México. Escuela Nacional de Enfermería y Obstetricia. Unidad de Investigación. Correo electrónico: gandhy_ponce@yahoo.com.mx

Cómo citar este artículo en edición digital: Jiménez López, E. E Ponce Gómez, G. (2019). Cuidando del embarazo: el caso de las parteras Tseltales en Chiapas, México. Cultura de los Cuidados (Edición digital), 23(53). Recuperado de http://dx.doi.org/10.14198/cuid.2019.53.19

Correspondencia: Gandhy Ponce Gómez. Calle 615. No. 18. Unidad Habitacional San Juan de Aragón. Delegación Gustavo A. Madero, C.P. 07979. Ciudad de México, México

Correo electrónico de contacto: gandhy_ponce@yahoo.com.mx Recibido: 23/10/2018; Aceptado: 11/01/2019
\end{abstract}

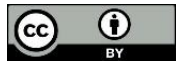

\section{ABSTRACT}

Justification: The existence of ethnic groups in Mexico, gives an important cultural significance for the care of pregnant women. In Sitalá, Chiapas, Mexico, midwives have built their trajectory through traditional generational knowledge, with a different way of treating maternal and child health problems with respect to the biomedical care system.

Objectives: Understand the cultural practices of midwives in the care of the pregnant woman of the Tseltal ethnic group.

Methodology: Ethnographic study of 8 participants, content analysis until saturation. Theoretical references of the Model Rachel Spector and Model of Cultural Competence of Larry Purnell.

Results and discussion: The sobada, is a manipulation of the traditional midwife used by the traditional health care system in indigenous communities of the Tzeltal ethnic group, they cure, accompany and advise in the primordial moments of family development.

Conclusion: Living midwifery provides accompanied care during pregnancy with safety, physical and emotional comfort. They are non-formal leaders of the communities and a means to generate changes in health; so the communication of the nursing professional is paramount in the conservation of health or self-care that can be achieved in these communities.

Keywords: Midwifery, cultural care, sobada.

\section{RESUMO}

Justificação: A existência do grupos étnicos no México, dá um importante significado cultural para o atendimento de 
mulheres grávidas. Em Sitalá, Chiapas, México, as parteiras construíram sua trajetória através do conhecimento geracional tradicional, com uma maneira diferente de tratar os problemas do saúde materna e infantil em relação ao sistema de cuidados biomédicos.

Objetivos: Compreender as práticas culturais das parteiras no cuidado da mulher grávida do grupo étnico Tseltal.

Metodologia: Estudo etnográfico do 8 participantes, análise de conteúdo até saturação. Referências teóricas do Modelo Rachel Spector e Modelo de Competência Cultural de Larry Purnell.

Resultados e discussão: A sobada, é uma manipulação da parteira tradicional utilizada pelo sistema tradicional do saúde em comunidades indígenas do grupo étnico Tzeltal, que cura, acompanha e aconselha os momentos primordiais do desenvolvimento familiar.

Conclusão: A enfermeira viva fornece cuidados acompanhados durante a gravidez com segurança, conforto físico e emocional. Eles são líderes não-formais das comunidades e um meio para gerar mudanças na saúde; então a comunicação do profissional do enfermagem é primordial na conservação da saúde ou autocuidado que pode ser alcançada nessas comunidades.

Palavras chave: Obstetrícia, cuidados culturais, sobada.

\section{RESUMEN}

Justificación: La existencia de etnias en México, da un significado cultural importante para la atención de embarazadas. En Sitalá, Chiapas en México, las parteras han construido su trayectoria a través de un saber tradicional generacional, con una forma distinta de tratar los problemas de salud materno-infantil con respecto al sistema de atención biomédico.

Objetivos: Comprender las prácticas culturales de las parteras en la atención de la embarazada de la etnia tseltal.

Metodología: Estudio etnográfico de 8 participantes, análisis de contenido hasta la saturación. Referentes teóricos del Modelo Rachel Spector y Modelo de competencia cultural de Larry Purnell.

Resultados y discusión: La sobada, es una manipulación de la partera tradicional utilizada por el sistema de cuidado a la salud tradicional en comunidades indígenas de la etnia tzeltal, ellas curan, acompañan y aconsejan en los momentos primordiales del desarrollo de las familias.

Conclusión: La partería vivida provee de cuidados acompañados durante el embarazo con seguridad, confort físico y emocional. Son líderes no formales de las comunidades y medio para generar cambios en la salud; así que la comunicación del profesional de enfermería es primordial en la conservación de la salud o el autocuidado que se pueda a lograr en estas comunidades.

Palabras clave: Partería, cuidado cultural, sobada.

\section{INTRODUCCIÓN}

En las zonas rurales del estado de Chiapas, México, las formas de vida, la situación social, cultural, política, económica y el cuidado de la salud son similares a muchos lugares de Latinoamérica, donde la presencia de diversas etnias da un significado cultural importante para la atención de las embarazadas, en el cual las parteras son pilares en la atención y cuidados. Específicamente el municipio de Sitalá tiene una población de 9887 personas que hablan alguna lengua indígena (tseltal), 
lo que representa $96 \%$ de la población. Para conocer como resuelven los problemas de salud que encuentran las parteras en su práctica cotidiana, es necesario escuchar de viva voz sus experiencias, sus prácticas de cuidados; el saber sobre su cultura, tradiciones, costumbres, formas de vida y en general la cosmovisión de las parteras como en el caso particular que nos ocupa, el de las parteras tzeltales.

De acuerdo con las estadísticas presentadas por el Instituto Nacional de Estadística Geografía e Informática, en México los estados con mayor porcentaje de partos atendidos por parteras son: Chiapas (74\%), Oaxaca (56\%), Guerrero (51\%), Tabasco (42\%), Veracruz (42\%), Puebla (33\%) y San Luis Potosí (27\%) (Instituto Nacional de Estadística Geografía e Informática, 1999).

Los primeros hechos en torno a la atención de la embarazada, se remontan a los inicios de la raza humana, su atención ha variado a lo largo de la historia y frecuentemente se concibe en un entorno lleno de mitos y tabúes (Alfaro, Villaseñor, Valadez, Guzmán y González, 2006a).

Los patrones culturales tradicionalmente han asignado a las mujeres el rol de curanderas, y ellas han sido también quienes se han encargado de la atención del parto, la cual se realiza en la casa de la embarazada y se encuentran referidos en múltiples registros (Alfaro, Villaseñor, Valadez, Guzmán y González, 2006b).

Así mismo, en relación a la atención del parto se encuentran muchos trabajos de investigación realizados en México y en el resto del mundo, pero en relación al cuidado de la salud de la embarazada muy poco se encuentra escrito sobre todo en lo que se refiere a las prácticas culturales.

Partera tradicional (PT) es la persona que asiste a la madre durante el parto y que ha adquirido sus conocimientos iniciales de partería por si misma o por aprendizaje con otras parteras tradicionales (Organización Mundial de la Salud, 1993). La NORMA Oficial Mexicana 007, define a la partera tradicional como a la persona que pertenece a comunidades indígenas y rurales y que ha sido formada y practica el modelo tradicional de atención del embarazo, parto, puerperio y la persona recién nacida, la cual se considera como personal no profesional autorizado para la prestación de los servicios de atención médica (Norma Oficial Mexicana NOM-007-SSA2-2016).

Las instituciones de salud deben comprender las costumbres, creencias y prácticas culturales de atención a la mujer en edad reproductiva, en estado de gravidez, en el parto, el puerperio y hasta el cuidado del recién nacido; de esta forma, no basta conocer cuáles son las prácticas culturales, es necesario que también sean admitidos en el sistema formal de salud para que puedan ser monitorizadas en todo momento, para saber si alguna partera tradicional está teniendo complicaciones con alguna embarazada y apoyarla, bien sea de forma física o con un vehículo para el traslado a algún hospital o unidad de salud con más capacidad resolutiva. De este modo eliminar paulatinamente los factores de riesgos asociados a los embarazos que se dan en condiciones de desigualdad.

En la revisión de la literatura se hace expreso que el cuidado del embarazo y el puerperio es asumido por los cuidadores culturales, las gestantes los visitan según el estado de salud y capacidad económica. Los Thewala se encargan del bienestar espiritual de la gestante mediante la realización de sobos, baños de armonización y tanteos. Las parteras son las responsables de atender el componente físico en la gestante, 
determinando la posición del bebé y realizando sobos para acomodarlo para el parto; así mismo, sugieren a la gestante el consumo de ciertos alimentos y el uso de plantas medicinales, tanto para infusión como baños (Muñoz, Castro, Chávez y Ortega, 2012).

En cuanto a las prácticas de cuidados del embarazo, inicialmente diagnostican el embarazo, mediante la palpación abdominal, percibiendo la frecuencia cardiaca fetal, además de la apreciación de un punto o prominencia palpada en el abdomen de las gestantes. Durante el control mensual realizan actividades de "tocada", que consiste en masajes sobre el vientre para hacer girar al bebé cuando este se encuentra en posición anormal, se recomienda a la embarazada que no debe realizar labores hogareñas relacionadas con hacer nudos, clavar puntillas, tapar un recipiente, pasar por debajo de las escaleras ya que ocasionan la trama, es decir, un parto difícil (López, Cataño, López, Velásquez, 2011).

En cuanto al uso de plantas medicinales en Veracruz, México las parteras comparten acerca de sus saberes ancestrales que existen plantas medicinales que son usados por parteras y médicos tradicionales para diversos padecimientos: citan al carrizo; para detener las hemorragias, la hierba del golpe; para la fertilidad femenina, la vergonzosa de hoja ancha; tratar la hemorragia posparto, el quelite blanco; para producir leche en mujeres, el zacate chichi; para prevenir el edema y fiebre posparto la, hierba del burro o zopilote (Gheno, Nava, Martínez y Sánchez, 2011).

En cuanto a las prácticas culturales, las mujeres perciben que se les facilita el proceso de parto, puesto que les brindan seguridad, apoyo emocional y físico a las gestantes, las parteras tradicionales preservan y promueven tradiciones que están arraigadas en las creencias culturales, con acciones como masajear el abdomen, enterrar la placenta en casa, orar y apoyar a la familia, cumpliendo así con las expectativas culturales de atención de la mujer, situación contraria a las prácticas institucionales de salud (Laza, 2015).

\section{REFERENTES TEÓRICOS}

Todo profesional de enfermería en diversos momentos de la práctica de cuidados se encontrará en contacto con diversas culturas. Debido al movimiento migratorio a causa de la globalización, personas de diversas regiones del mundo transitan por el territorio nacional; no obstante, solo en México tenemos una riqueza cultural inmensa. Rachel Spector en su modelo de Tradiciones en Salud, refiere que los profesionales de enfermería deberían ser el vínculo que traslade las creencias y prácticas del paciente al sistema de salud, y viceversa (Spector, 2002a). El Modelo de herencia cultural y tradiciones de salud de Rachel Spector menciona que esta teoría fue desarrollada por Estés y Zitzow en 1980, quienes pretendían describir hasta qué punto el estilo de vida de una persona refleja su cultura tradicional (Spector, 2002b). Esta teoría se ha ampliado con la intención de abarcar las diferentes culturas europeas, asiáticas, africanas o hispanas. Para Spector la consistencia hereditaria existe en un continuum, de manera que una misma persona puede poseer características de herencia (tradicional) o inconsistente (moderna o culturizada). Este concepto implica la determinación de los vínculos de una persona con sus antecedentes étnicos, culturales y religiosos (Spector, 2002c). 
Sugiere que el dispensador de cuidados para llegar a comprender lo que hace y que su práctica profesional resulte eficaz, debe tener las siguientes características:

- Ser culturalmente sensible, esto implica tener los conocimientos necesarios y comprender las tradiciones relacionadas con la salud y la enfermedad.

- Ser culturalmente congruente, significa que se debe tener la capacidad de llevar a la práctica los conocimientos que posen de forma adecuada $y$ rentabilizarlos desde el punto de vista de la enfermería mediante la dispensación de cuidados coherentes culturalmente y de gran calidad profesional.

- Ser culturalmente competente, se debe prestar atención de forma adecuada al paciente, considerando el contexto y manteniendo, por tanto, la filosofía holística de los cuidados.

Para desarrollar su modelo, Spector enfatiza la necesidad de realizar las siguientes valoraciones:

- Valoración del legado cultural: considera que debe ser valorado mediante el nivel de importancia de la cultura, la etnicidad y la religión en el individuo, la familia y la comunidad.

- Valoración de fenómenos culturales que afectan a la salud, se refiere a aspectos de orientación en el tiempo, espacio personal y territorialidad, comunicación, organización social, variables biológicas, hábitos de comida y alimentación, control del entorno (Spector, 2002d).

\section{Tradiciones en salud}

Cada individuo tiene el derecho a ser respetado por su singularidad y herencia cultural ya que ninguna cultura es mejor que otra sino diferente. La cultura ayuda a interpretarse a uno mismo y conocerse en cuanto al modo de responder en los cuidados proporcionados. Y la valoración de las tradiciones en salud es indispensable para conocer las conductas del cuidado que se practican y con ello poder brindar cuidados culturalmente adaptados por el profesional de salud (Siles, Cibanal, Vizcaya, Gabaldón, Domínguez \& et al., 2001a).

Valoración de las tradiciones de salud se integra por ocho dimensiones interrelacionadas: creencias y prácticas para el mantenimiento de la salud, creencias y prácticas para la protección de la salud, actividades realizadas con la restauración de la salud, salud y creencias tradicionales, salud y prácticas tradicionales, remedios tradicionales, salud y sanadores tradicionales, cuidados culturales y proceso de enfermería (Siles, Cibanal, Vizcaya, Gabaldón, Domínguez \& et al., 2001b). Con este modelo Rachel Spector resalta la importancia de tomar en cuenta lo que la gente piensa, siente, dice y hace, para cuidar o restaurar su salud, asimismo recomienda reflexionar al respecto e involucrarse para comprender el tipo de cuidado que la gente de cada cultura que se atiende necesita, considerando que cada cultura determina a sus miembros en su forma de pensar y actuar.

Cada cultura elabora su propia conceptualización de salud y de enfermedad, a partir de su cosmovisión, pero también de otras realidades sanitarias, y resuelve sus problemas desde su propia perspectiva, puntos de vista, capacidades y 
recursos (Siles, Cibanal, Vizcaya, Gabaldón, Domínguez \& et al., 2001c). En este sentido, se refuerza la necesidad de conocer, comprender e involucrarse con las diversas culturas que se atienden, para poder trabajar con un vínculo armonioso en torno al cuidado o restauración de la salud individual, familia y comunitaria.

\section{METODOLOGÍA}

Se trata de un estudio cualitativo que busca la descripción de la atención del embarazo por parte de parteras tradicionales en el municipio de Sitalá, Chiapas. El método etnográfico, permitió rescatar de forma efectiva las acciones, argumentos, entrevistas, observaciones y el sentir de las parteras tradicionales y con una observación participante. Fueron un total de 9 participantes entre los 52 y 76 años de edad, con una experiencia de partería en promedio de 25 años de práctica.

La fiabilidad y validez se estimó codificando 2 veces cada entrevista para analizando la coincidencia de códigos. Se realizó la contrastación de las percepciones propias y las dificultades de interpretación con expertos en la temática, hasta llegar a un nivel de acuerdo en la asignación de códigos e identificación de unidades de registro. Se llevó un registro etnográfico y el llenado de un diario de campo en 46 visitas con parteras y 38 visitas con parteras y mujeres embarazadas de la misma comunidad. Se trabajó con un traductor de Tseltal que ayudó a la traducción y verificación en la transcripción de narrativas. Este proyecto de investigación siempre considero el respeto a los derechos humanos de las parteras.

Se consideró la declaración de Helsinki y la ley general de salud, en los apartados correspondientes a las investigaciones cualitativas, así como la autorización del
Consentimiento Informado.

\section{RESULTADOS Y DISCUSIÓN}

Tras el análisis de contenido emerge la categoría:

- “Cómo va tu hijo?": se mencionan las formas de valoración que las parteras realiza para corroborar el embarazo, valorar el crecimiento y desarrollo fetal y para determinar el sexo fetal.

Quatrocchi, define a la sobada, no es un masaje cualquiera, sino una manipulación específica del cuerpo que permite a una particular concepción del organismo y de la salud, que forma parte del sistema medico local y del sistema ideológico más amplio (Quattrocchi, 2017).

Madrid y Castillo en 2001, refieren que el cuidado y la protección de la vida de las mujeres con una de las prioridades que se realiza a través de sobar el cuerpo (Madrid, Castillo, 2003).

Al realizar las visitas a las parteras del municipio de Sitalá, Chiapas, observamos que las parteras realizan esta maniobra a las embarazadas, generalmente a partir de los tres meses de edad gestacional o antes si existe dolor en alguna parte de estructura anatómica de la persona que solicita el cuidado, como se describe a continuación:

"Entonces a los tres meses ya le doy su sobadita y su té...es embarazo le digo, entonces ahora ya te voy a sobar" (Partera 2).

"Si esta con dolores, se le da un té ahora si no...no se le da nada, solo sus talladitas, solo su sobadita y ya. Sobar son los cuidados que le damos" (Partera 4).

En el quehacer de las parteras tradicionales "la sobada" forma parte fundamental de su cotidianidad. No 
obstante, considera ofrecer medidas de relajamiento en el cuerpo físico y emocional a las embarazadas, por lo cual les prepara una infusión de hierbas que ella cultiva en su patio para tener fácil acceso a ello (té de hierbas) procurando que el embarazo transcurra normalmente. La sobada, además de ofrecerse como terapia para la relajación, las parteras también la usan para aliviar molestias como el dolor, las náuseas y otros síntomas, que las embarazadas refieren durante la atención.

El estado de gravidez durante todo el proceso, transcurre por diversos momentos, las parteras argumentan que las mujeres acuden a ellas para llevar un control del embarazo, muchas veces las embarazadas refieren dolores abdominales; por lo que las parteras de acuerdo a su conocimiento optan por terapias de cuidado tradicionales para tratar molestias y dolores que consiste en sobadas, con la intención de aliviar las molestias que en ese momento padezcan. Las sobadas son masajes que aplican las parteras en las partes que dolorosas, siendo una actividad primordial en el cuidado del embarazo.

Las parteras tradicionales consideran que las sobadas son un recurso fundamental para el desarrollo de sus actividades, pasa a ser parte necesaria en su cotidianidad y como recurso comunitario para el cuidado de la salud materna. Hay ocasiones que acuden a ellas, mujeres que no saben si están embarazadas; las parteras mencionan que es necesario identificar y relacionar algunos aspectos de la vida que se han presentado en el organismo de la mujer, por ello, a través de una plática cordial, en el cual procuran establecer un clima de confianza con la mujer, le preguntan sobre la fecha en el que ha dejado de menstruar, si ha presentado nauseas o cualquier momento de malestar que sugiera un embarazo; para poder iniciar los cuidados y manejo de terapias para el control de estos malestares.

Mientras tanto, para la partera la sobada tiene diversos fines, el fin principal es el que va dirigido a aliviar las dolencias $y$ reestablecer el bienestar de la mujer, aliviando el cuerpo físico y las emociones de la embarazada; sin embargo, este procedimiento lleva consigo el interés de la partera por manifestarle la confianza que debe haber en ambas personas, en virtud de que la partera será su cuidadora por todo el proceso de embarazo, parto, puerperio y hasta el cuidado del recién nacido.

Se observó que también realizan la sobada a las embarazadas generalmente a partir de los tres meses de edad gestacional o antes si existe dolor en alguna parte de la anatomía de la persona que solicita el cuidado.

Sin embrago, las parteras también practican el manteado o manteada acompañado de sobadas como terapia para acomodar posiciones fetales anómalas, es una terapia que realizan con delicadeza, en la siguiente subcategoría se describen los detalles de esta terapia.

\section{CONCLUSIONES}

El análisis detallado y la interrelación entre estas categorías, permitió concluir que la magnitud y el impacto del cuidado de las parteras tradicionales en la mujer embarazada de las etnias mexicanas es fundamental dentro del desarrollo $y$ convivencia familiar mexicana, en específico de comunidades indígenas.

La participación de las parteras para el cuidado de la salud materna en el municipio de Sitalá, es muy importante para prevenir desenlaces lamentables durante toda la etapa del embarazo, entre las actividades 
que destacan para la práctica cultural de cuidados, las parteras tienen a su alcance diversas medidas terapéuticas para el control y seguimiento de algunas molestias que se presentan durante el embarazo. Las parteras inician la atención generalmente desde los dos meses de edad gestacional o antes si existe alguna molestia relacionada con el embarazo.

Entre las medidas terapéuticas, la sobada y el uso de infusiones de hierbas que en la mayoría de la ocasiones ellas mismas cultivan en su casa para tenerlos al alcance toda vez que se requiera; son de los procedimientos más usados por la mayoría de ellas, las parteras mencionaron que sobar son los cuidados que otorgan. Además de la sobada, la manteada o manteo es otro procedimiento que la partera realiza para acomodar la posición fetal transversa, oblicua o podálica, en la cosmovisión de las parteras de este municipio, atribuyen un poder mágico-curativo a la manta nueva o enahua de la madre.

Proposiciones a la luz del análisis de los hallazgos en el cuidado de las parteras de Sitalá:

"El manteado y la sobada propicios para acomodar la posición del bebé". Uso del A'ch pa'c (en lengua tseltal) para la salud de toda embarazada. Un masaje terapéutico particular realizado por las parteras de la etnia Tseltal con el fin de reposicionar un órgano o partes del cuerpo, y para nuestro caso, reposicionar al bebé, el cual se considerada momentáneamente "fuera de lugar". No es un masaje cualquiera, sino una manipulación específica del cuerpo que remite a una particular concepción del organismo y de la salud.
"Antes no estabas embarazada y ahora lo estás, tu cuerpo ha cambiado, se necesita reposicionar": "sobar", acomodar. Lo que forma parte del sistema del cuidado a la salud local y del sistema ideológico de la partera tzeltal y de otras regiones de México, siendo una práctica que requiere de un largo aprendizaje y un conocimiento profundo del uso de las manos realizado solo por personajes elegidos desde generaciones atrás.

Es así como los seres humanos activamente construyen su cultura en las relaciones sociales $\mathrm{y}$, a su vez, ella las moldea, coincidimos en que la partería vivida en la etnia tzeltal, cultura muy característica de Chiapas, México; provee de cuidados acompañados durante el embarazo y de los cuales participa muchas veces la familia y la partera como parte de dicha familia. Las proposiciones emanadas describes las dimensiones generales en que el cuidado heredado desde el pasado tzeltal llega a la contemporaneidad y permite disminuir la morbi mortalidad materno infantil.

La atención primaria a la salud debe estar fortalecida desde muchos ámbitos, los profesionales de enfermería debemos estar preparados para afrontar los retos que se van presentando en esta transición epidemiológica y con los cambios sociales actuales. Para fomentar y promover la salud materna mexicana de forma efectiva en las zonas más marginadas del país, es necesario contar con el apoyo de los diversos recursos comunitarios existentes; las parteras tradicionales son un apoyo importante en el desarrollo de nuestras actividades de cuidado, en virtud de que ellas ven a la atención de la salud materna en particular a la atención prenatal como su razón de ser, 
refieren que su labor es cuidar que el embarazo curse sin complicaciones, mediante diversos procedimientos $\mathrm{y}$ terapias alternativas.

De acuerdo a la concepción de cuidados que ellas tienen, se deben brindar y procurar los cuidados de acuerdo a la cosmovisión de las etnias, procurando que la partera sea un apoyo importante, en el que exista un vínculo armonioso y comunicación recíproca, puesto que es una aliada en el cuidado de la salud materna, en virtud de que ella realiza sus actividades con el enfoque cultural de la etnia a la que pertenece; para ello es necesario que olvidemos cualquier opinión preconcebida y negativa que se tenga sobre la atención que brindan las parteras tradicionales.

\section{BIBLIOGRAFÍA}

- Alfaro, A.N., Villaseñor, F.M., Valadez, F.I., Guzmán, S.A. \& González, T.Y. (2006) Algunos aspectos históricos de la atención al embarazo. Revista Investigación en Salud, 8 (1), 50-53.

- Gheno, H.A., Nava B.G., Martínez, C.A. \& Sánchez, V.E. (2011). Las plantas medicinales de la organización de parteras y médicos indígenas tradicionales de Ixhuatlancillo, Veracruz, México y su significancia cultural. Polibotánica. México, 31, 199-251.

- Instituto Nacional de Estadística Geografía e Informática (1999). Información Estadística del Sector Salud y Seguridad Social. Nacimientos por entidad federativa de ocurrencia según persona que atendió el parto. México: INEGI. Recuperado de http://www.inegi.gob.mx/prod_serv/contenidos/es panol/bvinegi/productos/continuas/sociales/salud/ 2003/cuaderno20a.pdf.

- Laza, V.C. (2015). Factores relacionados con la preferencia de las mujeres de zonas rurales por la partera tradicional. Revista Cubana de Salud Pública, 41(3), 487-496.

- López, L., Cataño, N., López, H. \& Velásquez V. (2011). Diversidad cultural de sanadores tradicionales afrocolombianos: preservación y conciliación de saberes. Aquichan, 11 (3), 287-304.

- Madrid, R.Z., Castillo, D. (2003). Investigación parteras y/o comadronas: "Prácticas culturales y sociales de las mujeres rurales Chortí en torno a la fertilidad y reproducción" Estudio de caso en Copán Ruinas. Rev. Población y Desarrollo, I, 79-88.

- Muñoz B.S., Castro E., Castro E.Z., Chávez N.N. \& Ortega R.D. (2012) Interculturalidad y percepciones en salud materno-perinatal, Toribio Cauca 2008-2009. Rev. Univ. Ind. Santander. Salud, 44 (1), 39-44.

- Norma Oficial Mexicana (2016). NOM-007-SSA22016. La atención de la mujer durante el embarazo, parto y puerperio, y de la persona recién nacida. Diario oficial de la federación, 7 de abril de 2016.

- Organización Mundial de la Salud. OMS/FNUAP/UNICEF. (1993). Parteras tradicionales, declaración conjunta. Ginebra: OMS. Recuperado de http://apps.who.int/iris/bitstream/10665/38859/1/9 243561502_spa.pdf.

- Quattrocchi, P. (2017). ¿Qué es la sobada? Elementos para conocer y entender una práctica terapéutica en Yucatán. Península, 1(2), 143-169. Recuperado de http://www.mayas.uady.mx/articulos/pdf/sobada. pdf.

- Siles, J., Cibanal L., Vizcaya, F., Gabaldón, E., Domínguez, J.M., et al., (2001). Una mirada a la situación científica de dos especialidades esenciales de la enfermería contemporánea: la antropología de los cuidados y la enfermería transcultural. Cultura de los cuidados, 5 (10): 72-87. Recuperado de https://rua.ua.es/dspace/bitstream/10045/4938/1/C C_10_11.pdf.

- Spector, R. (2002). Enfermería de cuidados culturales. Cultura de los cuidados; 6(11): 5-10. Recuperado de https://rua.ua.es/dspace/bitstream/10045/4857/1/C C_11_01.pdf. 